\title{
diffusion-înolarrentals
}

The Open-Access Journal for the Basic Principles of Diffusion Theory, Experiment and Application

\section{Transport Properties of Catalyst Supports Derived from a Catalytic Test Reaction}

\author{
Frank Friedel, Frank Janowski, Thomas Hahn, Dirk Enke
}

Universität Halle, Institut für Technische Chemie und Makromolekulare Chemie, Schlossberg 2, 06108 Halle, Germany, E-Mail: dirk.enke@chemie.uni-halle.de

\section{Introduction}

Porous solids with bimodal pore structures are widely used as catalyst supports. Examples are formed bodies, such as tablets, beads, extrudates, and monoliths, of molecular sieves, silica and alumina. The formed bodies are prepared usually by compacting fine micro- or mesoporous particles [1]. As a result, macropores are formed between the agglomerated particles. Recently, catalyst supports on the basis of silica and silica-alumina with hierarchical bimodal pore structures were prepared by phase separation in a sol-gel process [2]. Zhang et al. [3] prepared catalyst supports with bimodal pore structures by introducing silica, zirconia or alumina sol into the macropores of silica gel pellets.

In order to select optimal support material for a certain application, information regarding the transport characteristics of the micro/meso- and macropore system of the available bimodal catalyst supports is required. The transport parameters of the macropore system of a formed body can be determined using a Wicke-Kallenbach diffusion cell. Except for the well examined molecular sieves, there is only little information regarding the transport properties of the micro- or mesoporous primary pore system of catalyst supports [4].

Kotter et al. [5] proposed a procedure for the measurement of effective diffusivities of supported catalysts under reaction conditions. Here, the effective diffusivity is determined from reaction rate measurements. Additional information regarding the porosity and tortuosity factor of the catalyst support is necessary to obtain the pore diffusivity of a reactant under reaction conditions. The uniform distribution of the active component is another requirement.

A suitable model system is necessary to study the correlation between the texture properties (mean pore size) and transport characteristics (pore diffusivity) of the primary pore system of catalyst supports. The model catalyst support should be characterized by (i) a monomodal pore structure with pore sizes in the range between 2 and $20 \mathrm{~nm}$ (typical for the primary pore system of catalyst supports), (ii) a narrow pore size distribution and (iii) a flexible geometric form (beads and membranes with comparable texture properties).

\section{Results and Discussion}

In this work, porous glasses in shape of beads and ultrathin membranes with controlled mesoporosity in the range between 2 and $20 \mathrm{~nm}$ were used as catalyst supports. The ultrathin membranes are characterized by comparable texture properties to 
the support beads. The hydrogenation of benzene over nickel catalysts on the basis of the porous beads was used to investigate the transport characteristics of the mesopores inside the primary particles of silica supports. The nickel supported catalysts were prepared by equilibrium adsorption in $\mathrm{Ni}\left(\mathrm{NO}_{3}\right)_{2}$ aqueous solution. Strong metal-support interactions in combination with an optimised washing procedure led to a uniform distribution of the active component. The characteristic texture and transport properties of the supports were retained after the modification procedure. The effective diffusivity of benzene under reaction conditions was determined from the point of intersection of the lines separating the kinetic from the mass transfer controlled region in the Arrhenius plots according to [5]. The tortuosity factors of the supports were obtained from measurements of the permeability of nitrogen at $25^{\circ} \mathrm{C}$ and 1.1 bar through the corresponding membranes.

Except for the smallest pore size $(2 \mathrm{~nm})$, the mass transfer in all model systems was in the Knudsen region under the experimental conditions. The tortuosity factors of the ultrathin porous glass membranes varied between 1.5 and 40 depending on the pore diameter and the porosity of the membrane. The pore diffusivity of benzene under reaction conditions was estimated by $D_{P(\text { benzene })}=\tau_{\text {(membrane) }} \cdot D_{\text {eff(benzene) }} / \varepsilon_{\text {(beads })}$. The pore diffusivities obtained were in the range between $1 \cdot 10^{-6}$ and $2 \cdot 10^{-5} \mathrm{~cm}^{2} / \mathrm{s}$. There was a linear correlation between the pore diffusivity and the mean pore diameter of the support in the investigated pore size range. The low absolute values in contrast to theoretical calculations (Knudsen diffusivity of benzene at reaction temperature in a straight cylindrical pore with the corresponding pore diameter) may arise from strong interactions of the diffusing reactant with the surface hydroxyl groups of the support. Pore roughness effects are another possible explanation.

\section{Conclusion}

Porous glasses in shape of beads and ultrathin membranes with comparable texture properties are an ideal model system to investigate the transport characteristics of the mesopores inside the primary particles of silica supports. The hydrogenation of benzene over nickel catalysts based on the porous glass beads is a suitable test reaction to estimate the effective diffusivities. The tortuosity factors can be obtained from measurements of the permeability of membranes with comparable texture properties. Now, the pore diffusivity of benzene under reaction conditions can be calculated.

\section{References}

[1] U. Hammon, M. Kotter, Chem.-Ing.-Tech. 56 (1984) 455 - 463.

[2] N. Nakamura, R. Takahashi, S. Sato, T. Sodesawa, S. Yoshida, Phys. Chem. Chem. Phys. 2 (2000) 4983 - 4990.

[3] Y. Zhang, Y. Yoneyama, K. Fujimoto, N. Tsubaki, Journal of Chemical Engineering of Japan 36 (2003) 874 - 880.

[4] T. Dogu, Ind. Eng. Chem. Res. 37 (1998) 2158 - 2171.

[5] M. Kotter, P. Lovera, L. Riekert, Ber. Buns. Physik. Chemie 80 (1976) 61 - 66. 